\title{
The Prevalence of Phenylketonuria in Arab Countries, Turkey, and Iran: A Systematic Review
}

\author{
Ashraf El-Metwally $\mathbb{D}^{1},{ }^{1,2}$ Lujane Yousef Al-Ahaidib, ${ }^{3}$ \\ Alaa Ayman Sunqurah, ${ }^{1}$ Khaled Al-Surimi, ${ }^{1}$ Mowafa Househ, ${ }^{1}$ Ali Alshehri, ${ }^{1}$ \\ Omar B. Da'ar, ${ }^{1}$ Hira Abdul Razzak, ${ }^{4}$ and Ali Nasser AlOdaib ${ }^{3,5}$

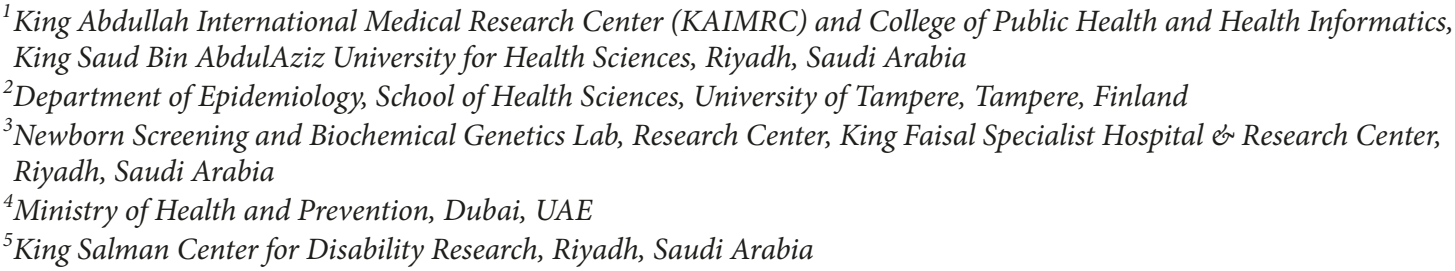 \\ Correspondence should be addressed to Ashraf El-Metwally; elmetwally.ashraf@outlook.com
}

Received 18 December 2017; Revised 13 February 2018; Accepted 4 March 2018; Published 18 April 2018

Academic Editor: Peter J. Oefner

Copyright (c) 2018 Ashraf El-Metwally et al. This is an open access article distributed under the Creative Commons Attribution License, which permits unrestricted use, distribution, and reproduction in any medium, provided the original work is properly cited.

Background/Objectives. This paper seeks to identify the prevalence of Phenylketonuria (PKU) in Arab countries, Turkey, and Iran. The study reviewed the existence of comprehensive national newborn screening programs and reported consanguinity rates. Methods. A computer based literature search was conducted using relevant keywords to retrieve studies conducted on PKU. A total of 34 articles were included. Prevalence was categorized based on the type of screening method used for PKU diagnoses. Results. The prevalence of classical PKU diagnosed through a comprehensive national newborn screening program ranged from $0.005 \%$ to $0.0167 \%$. The highest prevalence was reported in Turkey at $0.0167 \%$, whereas the lowest prevalence was reported in the UAE, $0.005 \%$. Conclusion. The findings of this review emphasize the need for the establishment of more efficient reporting systems in these countries that would help measure Disability-Adjusted Life Year (DALY) in order to estimate the overall societal burden of PKU.

\section{Introduction}

Phenylketonuria (PKU) (OMIM 261600) is an autosomal recessive inborn error of phenylalanine (Phe) metabolism, occurring in approximately $1: 15,000$ people. PKU is mainly caused by a deficiency of phenylalanine hydroxylase (PAH; 612349), the enzyme that catalyzes the hydroxylation of phenylalanine to tyrosine [1]. Hyperphenylalaninemia can also be due to defects in the regeneration or biosynthesis of the enzyme cofactor tetrahydrobiopterin (BH4). If untreated or undiagnosed, the neurotoxic effects of excess phenylalanine can lead to impaired postnatal cognitive development. Both types of hyperphenylalaninemias (PAH and $\mathrm{BH} 4$ deficient) are thought to be heterogeneous disorders that vary from severe, for example, classical phenylketonuria (PKU), to mild, benign, and transient forms. Enzyme deficiency yields a spectrum of disorders such as mild hyperphenylalaninemia, mild phenylketonuria, and classic phenylketonuria. Classic phenylketonuria is a result of near complete or complete deficiency of phenylalanine hydroxylase activity which will lead to profound and irreversible intellectual disability in the absence of dietary restriction of phenylalanine. Mild hyperphenylalaninemia and mild phenylketonuria are linked to a lower risk of impaired cognitive development if left untreated [2]. All of the aforementioned are included in OMIM 261640 with cytogenetic location: 12q23.2. Deficiency of $\mathrm{BH} 4$ is a rare disorder that changes the levels of various substances in the body, including phenylalanine [3]. Clinical manifestations of $\mathrm{BH} 4$ deficiency include intellectual disability, neurological deterioration, difficulty swallowing, 
TABLE 1: Incidence of PKU by populations. Source: $[11,13,14]$.

\begin{tabular}{lcc}
\hline Regions & Countries & Incidence of PKU \\
\hline \multirow{3}{*}{ Asian populations } & China & $1: 17,000$ \\
& Korea & 1 in 41,000 \\
& Japan & 1 in 125,000 \\
\hline & Ireland & 1 in 4,500 \\
& Scotland & 1 in 5,300 \\
Curopean populations & Hungary & 1 in 7,000 \\
& Denmark & 1 in 11,000 \\
& France & 1 in 12,000 \\
& Norway & 1 in 13,500 \\
& United Kingdom & 1 in 14,500 \\
& Italy & 1 in 17,000 \\
North America & Finland & 1 in 200,000 \\
\hline Oceania & United States & 1 in 10,000 \\
& (Caucasians) & 1 in 22,000 \\
\hline & Canada & 1 in 10,000 \\
\hline
\end{tabular}

movement disorders, behavioral problems, seizures, and an incapability to control body temperature.

Today, the clinical manifestations of classic phenylketonuria are rarely reported in the developed countries, where newborn screening (NBS) is prevalent. NBS has permitted the early detection and successful treatment with diet low in phenylalanine. The first NBS program emerged in the United States in the early 1960s [4] and became universal in most developed countries [5]. With the use of state-of-the-art detection methods such as tandem mass spectrometry, PKU can be diagnosed readily in blood specimens collected by heel-prick from newborns, 24 hours of age, and spotted onto a filter paper that contains all their demographic information [6].

Newborn screening is the principal population-based public health screening program which is being practiced at present across the globe [7]. In case of PKU, it was found that the prevalence differs between different populations [8]. The incidence of PKU varies among ethnic groups and geographic regions worldwide [9]. For example, Caucasians are effected at a rate of $1: 10,000$ birth in the United States [10]. In Europe, the highest incidence has been observed in Ireland at a rate of $1: 4,500$ [11]. It is also common in few parts of China, while it is rarely observed in African nations. In Turkey, an incidence as high as 1:2,600 has been reported [12]. Supplementary information about incidence rates in different countries is mentioned in Table 1 [12-14]. Given its autosomal recessive inheritance, consanguinity among carrier couples is considered as the main risk factor for PKU [3].

This review aims to investigate the prevalence and incidence of PKU in Arab countries, Turkey, and Iran, which share similar culture and customs. This study will also explore the role of NBS programs in estimating PKU prevalence and incidence.

\section{Method}

Electronic search using Pub Med, Embase, and Google Scholar was conducted to extract articles addressing the epidemiology of Phenylketonuria in Arab countries, Turkey, and Iran. Key words used for our search included Phenylketonuria or PKU or aminoacidopathies or metabolic disorders or inborn error metabolism and (prevalence or incidence or frequency) and (newborn screening program or selective screening or national neonatal screening or tandem mass spectrometry) and (Saudi or Kuwait or Oman or United Arab Emirates or Bahrain or Qatar or Egypt or Iraq or Syria or Jordon or Sudan or Libya or Tunisia or Algeria or Morocco or Palestine or Lebanon or Yemen).

2.1. Inclusion and Exclusion Criteria. Inclusion criteria were English language articles published in peer-reviewed journals from January 1982 to December 2017, studies focusing on prevalence/incidence of PKU and NBS programs in the countries mentioned above. Any case reports/series or articles tackling treatment, molecular mutation, and molecular diagnosis were excluded.

2.2. Selection and Data Extraction. Comprehensive search terms such as Phenylketonuria or PKU or aminoacidopathies or metabolic disorders or inborn error metabolism were systematically applied along with Boolean operators. A broad search of Pubmed and Embase databases yielded 2487 records. After removing duplicate records, a total of 1772 were identified in our search, of which, 1702 were irrelevant and were excluded based on title/abstract screening. Finally, 70 full-text articles were assessed for eligibility and were screened against the inclusion criteria (including 18 Arab countries). A total of 48 articles were further eliminated because PKU prevalence/incidence was neither mentioned nor were the researchers able to extract data or self-calculate prevalence. A secondary search was performed by crossreferencing and using the same keywords in Google Scholar that resulted in a total of (12) articles, which did not appear in our original PubMed and/or Embase search. Consequently, the total number of articles included in this systematic review was 34 . The review was conducted by two professionals in the field of epidemiology and public health. Any disagreements between the two researchers were solved by consensus.

\section{Results}

A total of 34 prevalence/incidence studies conducted in different years and regions were included in this review (see Table 2). In some of the prevalence/incidence studies, prevalence was self-calculated in 9 articles and corrected in 11 articles. Prevalence/incidence studies were further categorized to the type of study whether it was a national NBS program ( $n=5$ articles) [6, 15-18], regional/governorate NBS program ( $n=6$ articles) [19-24], selective screening for newborns ( $n=6$ articles) [25-30], selective screening of sick/symptomatic newborns and/or infants, children, and adults ( $n=9$ articles) [31-39], selective screening for both newborns and sick/symptomatic newborns and/or infants, 


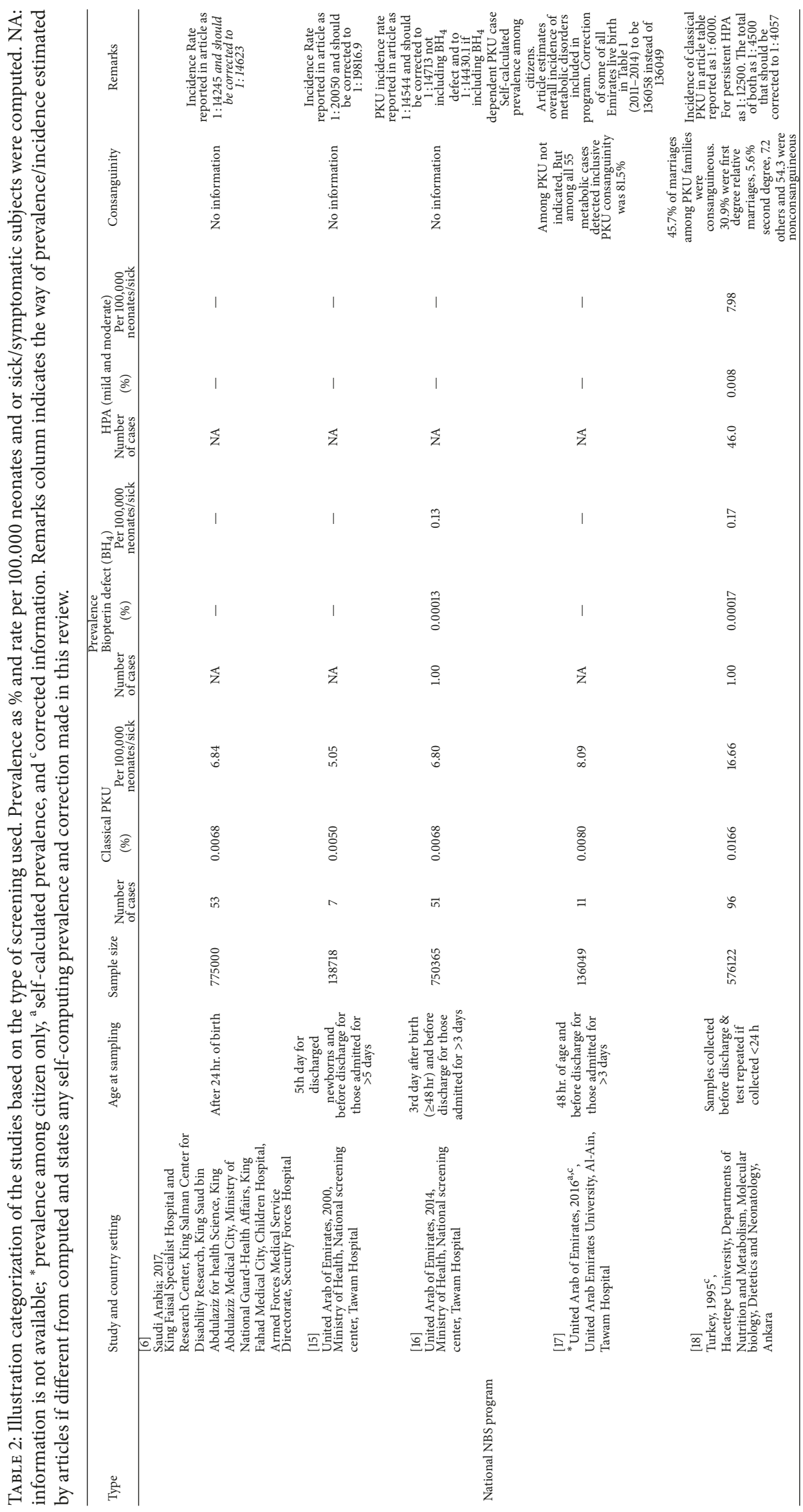




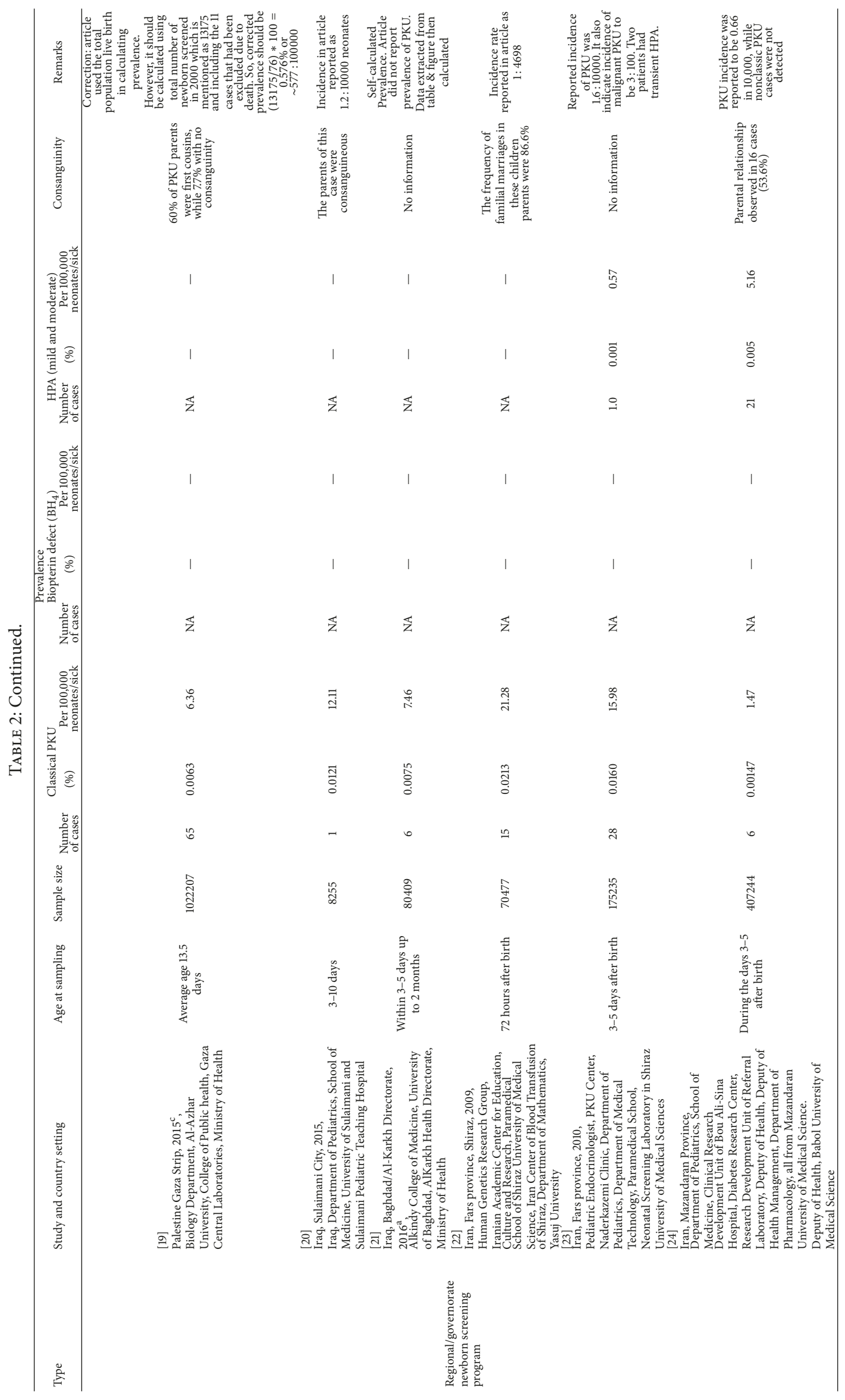




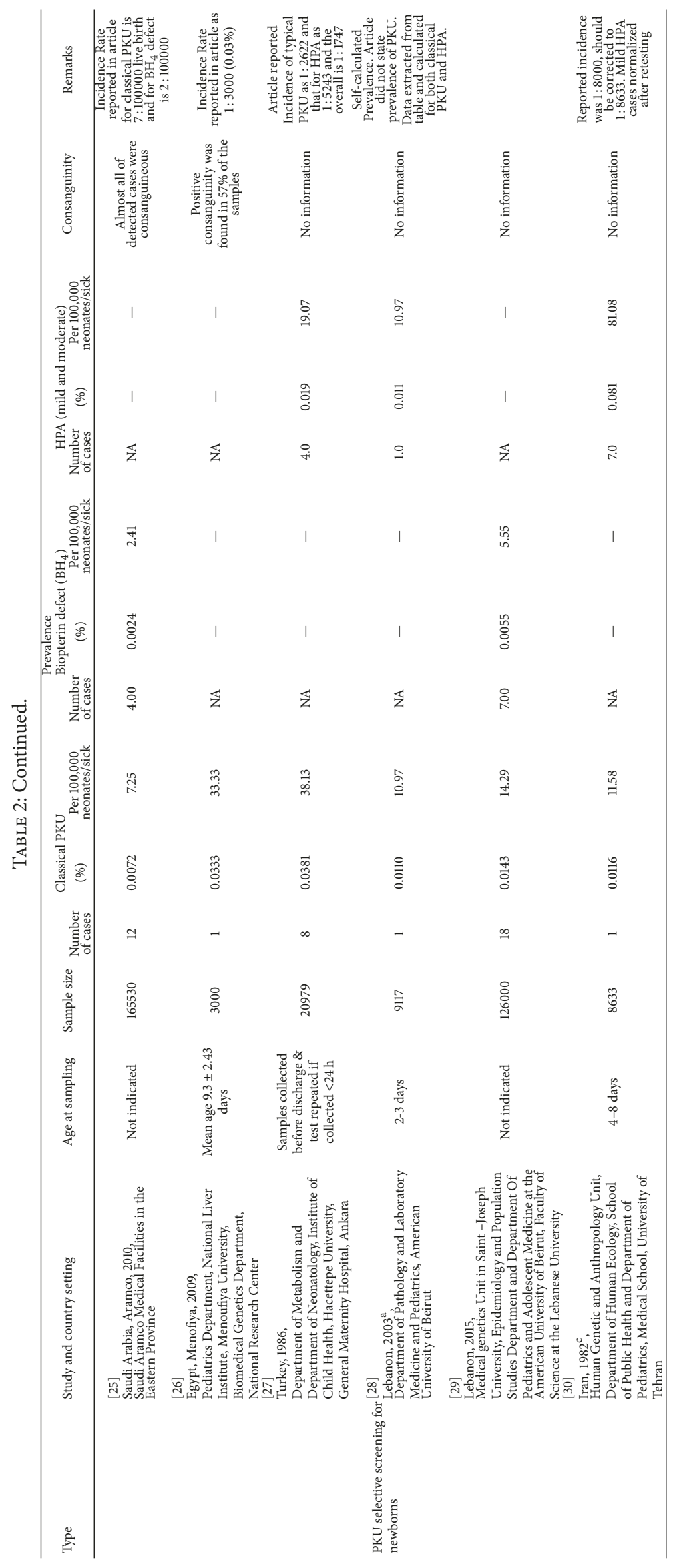




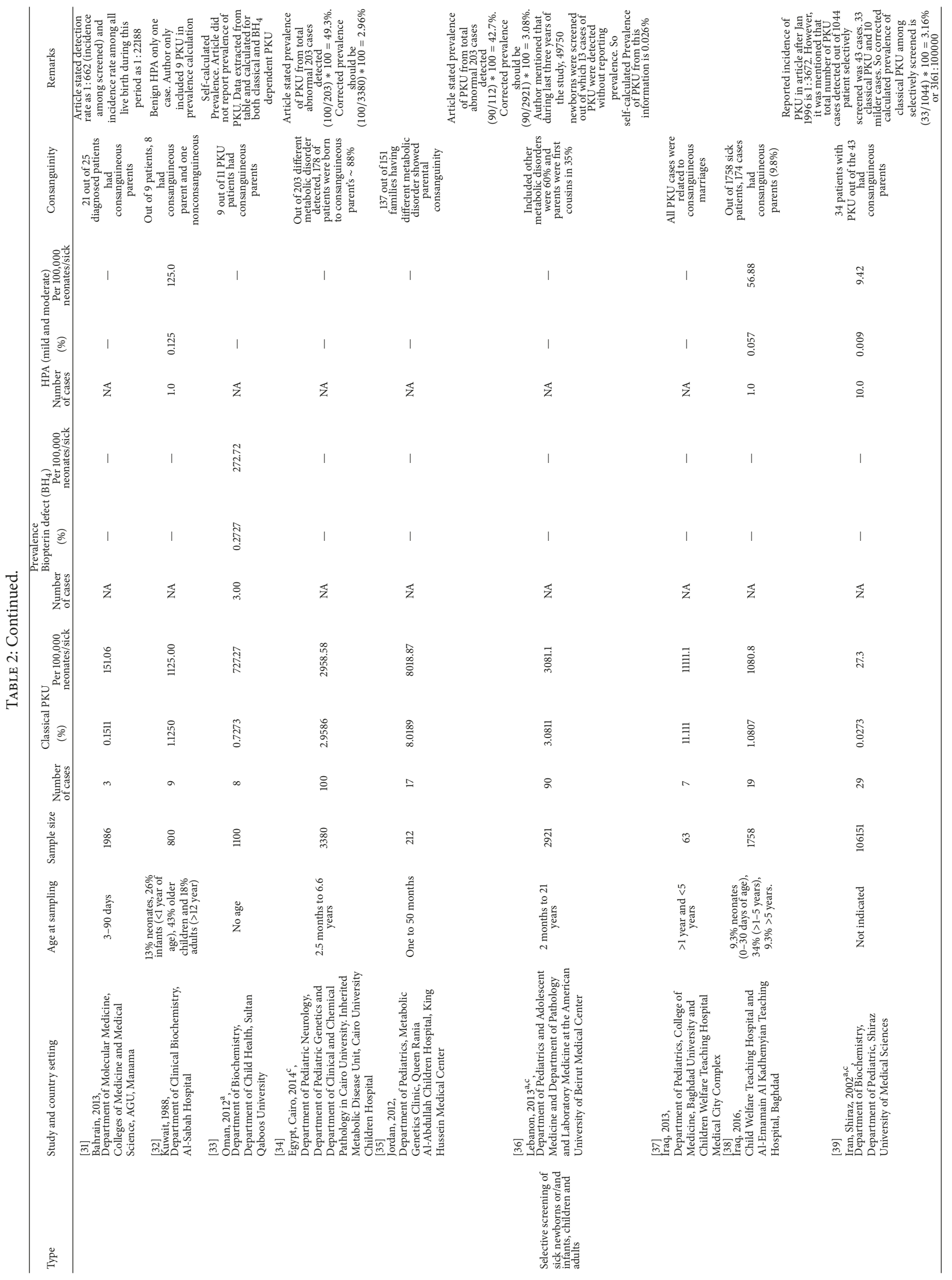




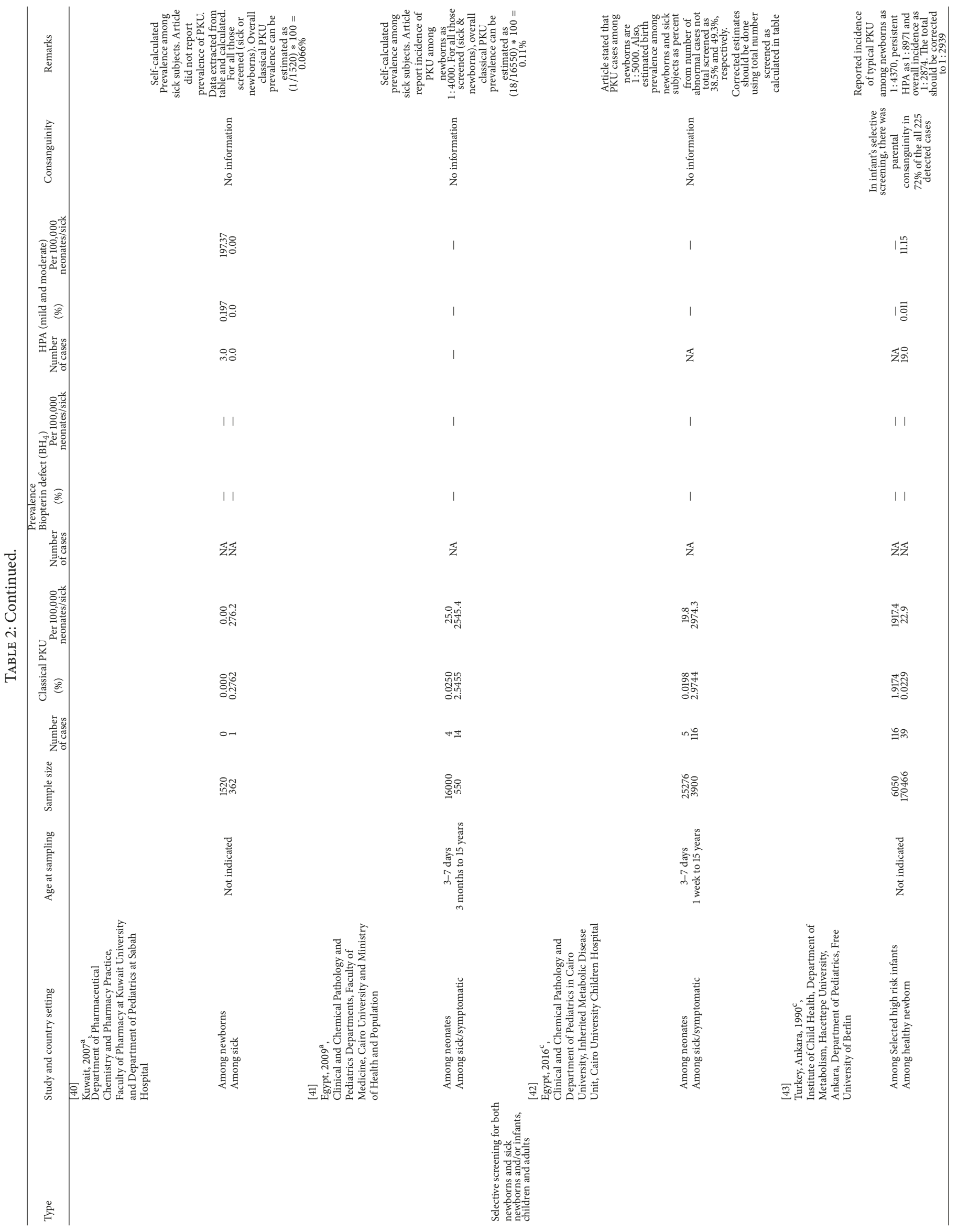




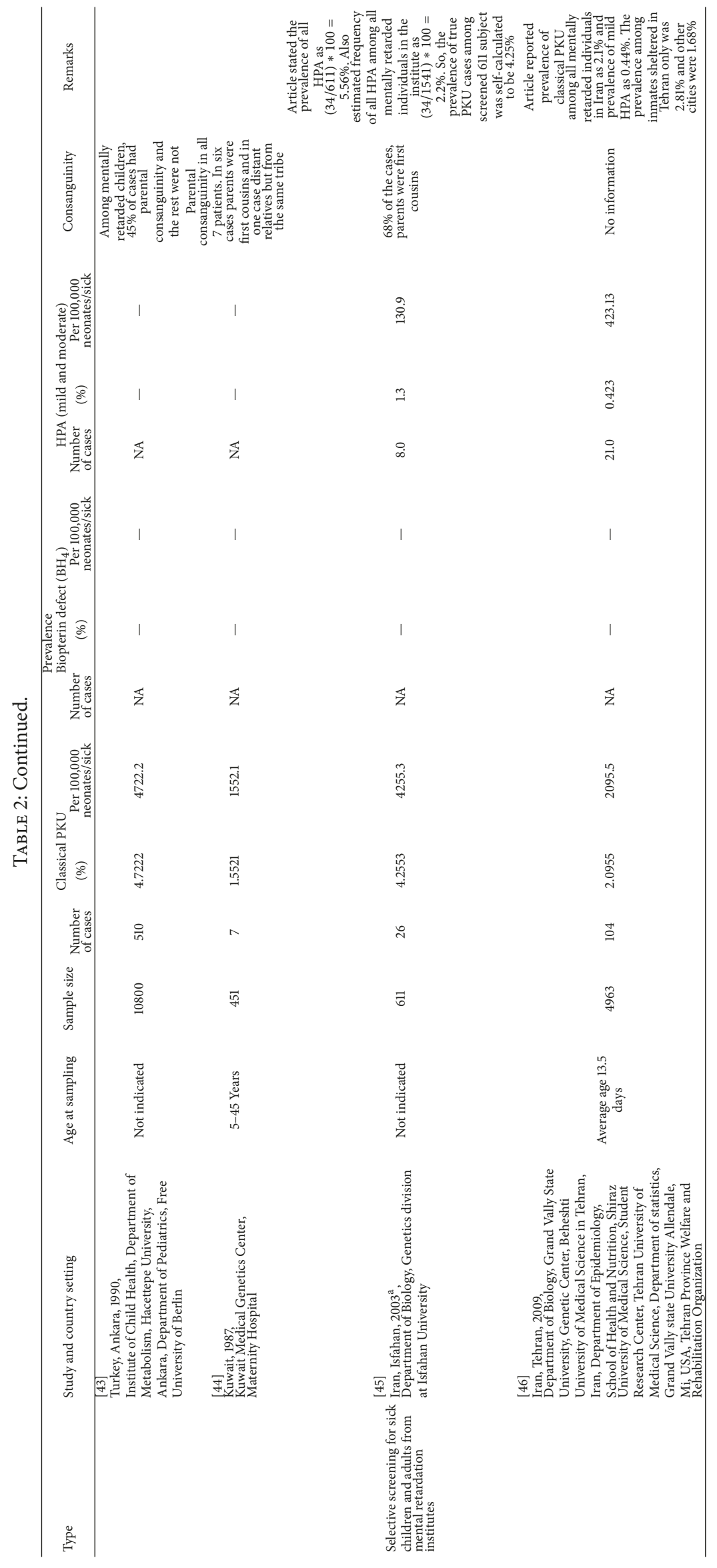


children, and adults ( $n=4$ articles) [40-43], or selective screening for sick/symptomatic children and adults from institutions for mentally challenged ( $n=3$ articles) [4446]. In addition, a study conducted in Turkey addressed PKU prevalence among newborns, sick/symptomatic subjects, and mentally challenged individuals. For all selected studies, prevalence of classical PKU, BH4 dependent PKU, and mild-moderate HPA were calculated as a percentage, and as a rate per 100,000 neonates/population, also presented in Table 2 [43]. Furthermore, consanguinity rate is indicated wherever available.

To conduct a reliable comparison of PKU prevalence, and as most of the studies reported prevalence and/or incidence in different ways, we first sought to unify the prevalence calculation in the form of percentage and rate per 100,000 of the screened population. Then, we categorized the studies by the population used to estimate prevalence into either national, regional, and selective screening programs or studies conducted in institutions for the mentally challenged. Moreover, a comparison was conducted using classical PKU prevalence as the most severe form in addition to the fact that not all (only few studies) gave estimates for $\mathrm{BH} 4$ dependent PKU (6 studies) and mild/moderate HPA (13 studies). Prevalence of self- calculation for PKU (including classical type or BH4 dependent PKU and HPA) was generated by extraction of the available information from articles included in this study by dividing the number of cases by the number of life births or sample size in the study during a specific year. Prevalence calculations were tabulated and expressed as percentage or as rate per 100,000 of population screened. Though we calculated PKU prevalence for all studies considered, only national programs will yield solid estimates.

Prevalence of classical PKU extracted or self-calculated from articles using comprehensive national NBS programs ranged between $0.005 \%$ and $0.0167 \%$. The corresponding range for regional/governorate NBS programs was $0.0015 \%$ to $0.0213 \%$. Selective screening programs of newborns gave prevalence of $0.0072 \%$ to $0.0381 \%$. However, in articles estimating PKU via selective screening of sick/symptomatic subjects [newborns, infants, children, and adults], the prevalence was reported to be between $0.0273 \%$ and $11.1 \%$. Prevalence in institutions caring for mentally challenged individuals ranged from $1.55 \%$ to $4.722 \%$. Four articles reported prevalence based on selective screening of both apparently healthy newborns and sick/symptomatic newborns (i.e., neonates who have missed newborn screening, thus, becoming symptomatic or acting abnormally in any way). Prevalence for selectively screened newborns was $0.0198 \%-0.0250 \%$ and prevalence for sick/symptomatic subjects ranged from $1.917 \%$ to $2.974 \%$.

\section{Discussion}

The review addressed and sought to shed light on the epidemic of PKU in the Arab countries, Turkey, and Iran. To our knowledge, this is the first systematic review conducted to summarize the prevalence of PKU in these countries. Despite the lack of published data on PKU prevalence in many Arab countries such as Algeria, Syria, Libya, Sudan, and Yemen, most likely due to the absence or limitation of comprehensive screening programs [47], our review still reflects the high prevalence of PKU in Saudi Arabia, United Arab Emirates (UAE), Turkey, Gaza Strip, Sulaimani, the Baghdad region in Iraq, and the Fars region in Iran. Our results show that prevalence of classical PKU in countries having national newborn screening programs ranges from $0.005 \%$ to $0.0167 \%$. The highest prevalence was reported for Turkey in $1995(0.0167 \%)$ [18] and the lowest one for the UAE in $2003(0.005 \%)$ [15]. In regions conducting NBS, prevalence ranged from $0.0015 \%$ in the Mazandaran Province [24] to $0.02 \%$ in the Fars region in south-central Iran [22].

The prevalence of classical PKU among selective NBS studies ranged between $0.0072 \%$ and $0.038 \%$. The lowest prevalence was reported for the Aramco Province in Eastern Saudi Arabia (0.0072\%) [25] and the highest for Ankara $(0.038 \%)$ [27]. Other studies estimated prevalence through selective screening for sick/symptomatic newborns and/or infants, children, and adults such as the ones conducted in Bahrain, Kuwait, Oman, Egypt, Jordan, Lebanon, and Iran. PKU prevalence among sick/symptomatic newborns was highest in the Jordan study (8\%) [35] (due to relatively a small sample size) and the lowest in the study was conducted by Golbahar et al. with $0.0273 \%$ [31]. Among mentally challenged individuals, the highest prevalence was noted in Turkey during 1990 (4.722\%) [43] and the lowest in Kuwait (1.55\%) [44]. Prevalence among sick/symptomatic subjects was the highest in Egypt during 2009 (2.5\%) and the lowest in Turkey in 1990 (0.02\%).

To date, only a few countries such as Saudi Arabia, UAE, Qatar, and Turkey in the region have implemented comprehensive national NBS programs with relatively high coverage that aim for early detection of PKU along with other treatable disorders in an attempt to reduce disability rates. The percentage uptake (or coverage) of newborn screening in the UAE was increased from $50 \%$ in 1998 to reach $95 \%$ in 2010, with a prompt increase in the year 2003 [16]; however, these levels are still below the international coverage standards (99\%) [48].

Unfortunately, our search failed to find any published data showing the prevalence of PKU in Qataris. The prevalence of PKU in Saudi Arabia was $0.0068 \%$. In UAE, PKU prevalence was $0.0081 \%$ analogous to the prevalence $(\sim 0.0073 \%)$ for the Aramco Province in Eastern Saudi Arabia. Consecutive studies on PKU prevalence in UAE have demonstrated an increase in prevalence with time from $0.005 \%$ in 2003 to 0.0068 in 2014, and finally $0.008 \%$ in 2016 .

A global comparison of incidence rates between countries with nationwide NBS programs shows that Japan, among Asian countries, reports the lowest rate with 1:125,000, whereas incidence in China is $1: 17,000$ [10]. Saudi Arabia is close to the PKU incidence of Japan at 1:14,623 [6] and UAE $1: 12,369$ [17]. On the other hand, the incidence rate among Caucasians in North America $(1: 10,000)$ is lower than those reported for Japan and China [49]. A similar study give reported incidence for Australia [10]. Among European countries, incidence rates among Saudis are higher than the rate of 1:4,500 reported for Ireland [11] but comparable to rates recorded in Denmark 1:12,000, France 1:13,500, Norway $1: 14,500$, and finally UK $1: 14,300[10]$. 
The consanguinity rate is very high in Arab countries as reported by most of the articles in our search. For example, 9 out of 11 PKU patients in Oman [33] and 8 out of 9 PKU patients in Kuwait had consanguineous parents [32]. Other studies conducted in Iraq [37] indicated that all 7 cases detected with PKU had consanguineous parents. These findings were consistent with previous studies where $57 \%$ of PKU patients in Egypt [26], 60\% of PKU cases in Gaza Strip [19], 86.6\% of PKU patients in Iran [22], and 34 patients out of 43 PKU cases from Iran [31] had consanguineous parents. Similarly, a recent study conducted in 2017 in Mazandaran Province in Iran indicated that parental family relationships among confirmed PKU cases were 53.6\% [24]. Congruently, another study from Iraq, Sulaimani city [20], reported only one case diagnosed with PKU being a product of consanguineous parents.

Addressing some recent articles, consanguinity rates among all cases with different metabolic disorder including PKU were reported. For example, a Jordanian study [35] reveals that out of 151 families, 137 cases had parental consanguinity. Similarly, Al-Jasmi et al. [17] in UAE declared that, among all metabolic disorders detected including PKU, consanguinity was $81.5 \%$. Alternatively, Selim et al. [34] showed that $88 \%$ of patients were born to consanguineous parents in Egypt. These results concur with Moammar et al. [25] findings in Saudi Arabia revealing all detected cases to have consanguineous parents. A study conducted in Gaza in contrast confirmed that some PKU cases were not consanguineous [19]. Nevertheless, it fails to mask the fact that most of the studies reporting the cases arose from consanguineous marriages.

\section{Limitations}

There were certain limitations to this review. First, our search was limited to publications in English. However, most if not all research conducted in the Arab world is published in English. One major drawback was attributed to the study design itself, in particular for prevalence/incidence studies where data from most of the articles were based on retrospective data collection either from medical records or registries. This kind of routine data has its own disadvantages such as incompleteness or inaccurateness. Other limitations include small sample size (63 samples from sick/symptomatic children in Iraq) in Rabab Thijeel study [37]. There is still an ambiguity with regard to the high prevalence of PKU (11.1\%) that cannot be generalized. Likewise, another possible limitation involved the way prevalence/incidence calculations were reported in some studies, where some articles used the denominator as a number of all live births during the study period and not the actual number of screened subjects. Others perform PKU estimations by using a total number of abnormal cases as a denominator instead of total number screened. For those incorrect estimates, corrections were made and documented in Table 2.

\section{Implications for Future Research}

PKU if not detected and treated early will lead to disability which presents a great socioeconomic burden for any country. Unfortunately, only few countries in the region including Saudi Arabia, UAE, Qatar, and Turkey have established active and comprehensive national NBS programs for PKU along with other disorders. More studies are needed in the region to monitor and study PKU. At the public level, and since consanguinity is the main factor of having the disorder in our region, continuous awareness campaigns through media, schools, and universities are recommended to educate the public about potential health risks posed by marriage between close relatives. Genetic counselors also play a big role in educating and helping the parents and affected siblings in not having another affected child during future pregnancy by introducing them to primary prevention such as prenatal diagnosis or Preimplantation Genetic Diagnosis (PGD). Issuing a policy through governments to mandate the screening test for every newborn is one effective approach to reduce PKU. Due to the rarity of specialized experts in this field, physicians, scientist, lab technologist, and governments should support training programs to compensate for this inadequacy.

\section{Conclusion}

In light of this review, our search demonstrated the need for establishment of more research work so as to investigate the true prevalence of PKU in our region using comprehensive population screening tests. The data in regard to prevalence, follow-up, and identification of other possible risk factors or other disease spectrum associated with PKU is scarce in our region. Our research through PubMed, Embase, and Google Scholar failed to find published data about reliable or recent PKU prevalence in many Arab countries such as Syria, Yemen, Libya, Morocco, Algeria, Tunisia, and Sudan.

Future research should also focus on measuring the Disability-Adjusted Life Year (DALY) to demonstrate overall burden of this disease as well as other genetic diseases. Estimating DALY is another successful measure to estimate years of life lost due to premature mortality (YLL) and years of life lived with disability (YLD). Providing such data will definitely give true estimates of this problem and allow for effective intervention programs to reduce disease burden.

\section{Conflicts of Interest}

The authors declare that there are no conflicts of interest regarding the publication of this article.

\section{Acknowledgments}

This project was funded by King Abdullah International Medical Research Center (KAIMRC), Riyadh, Saudi Arabia.

\section{References}

[1] N. Blau, F. J. Van Spronsen, and H. L. Levy, "Phenylketonuria," The Lancet, vol. 376, no. 9750, pp. 1417-1427, 2010.

[2] I. Følling, "The discovery of phenylketonuria," Acta Paediatrica, vol. 83, pp. 4-10, 1994. 
[3] N. Blau, M. Duran, K. M. Gibson, and C. Dionisi Vici, Physician's Guide to the Diagnosis, Treatment, and Follow-Up of Inherited Metabolic Diseases, Springer, Berlin, Germany, 2014.

[4] R. Guthrie and A. Susi, "A simple phenylalanine method for detecting phenylketonuria in large populations of newborn infants," Pediatrics, vol. 32, pp. 338-343, 1963.

[5] L. L. McCabe, B. L. Therrell Jr., and E. R. B. McCabe, "Newborn screening: Rationale for a comprehensive, fully integrated public health system," Molecular Genetics and Metabolism, vol. 77, no. 4, pp. 267-273, 2002.

[6] M. Alfadhel, A. Al Othaim, S. Al Saif et al., "Expanded newborn screening program in saudi arabia: incidence of screened disorders," Journal of Paediatrics and Child Health, vol. 53, no. 6, pp. 585-591, 2017.

[7] V. A. Moyer, N. Calonge, S. M. Teutsch, and J. R. Botkin, "Expanding newborn screening: process, policy, and priorities," Hastings Center Report, vol. 38, no. 3, pp. 32-39, 2008.

[8] P. Hardelid, M. Cortina-borja, A. Munro et al., "The birth prevalence of PKU in populations of European, South Asian and Sub-Saharan African ancestry living in South East England," Annals of Human Genetics, vol. 72, no. 1, pp. 65-71, 2008.

[9] P. Moradi, B. Sari-Sarraf, Z. Khamnian et al., "Occurrence of phenylketonuria in the world: a systematic review and metaanalysis," Tașvìr-i salāmat, vol. 6, no. 4, pp. 1-2, 2016.

[10] R. A. Williams, C. D. Mamotte, and Burnett. J. R., "Phenylketonuria: an inborn error of phenylalanine metabolism," The Clinical Biochemist Reviews, vol. 29, no. 1, p. 31, 2008.

[11] A. G. DiLella, S. C. M. Kwok, F. D. Ledley, J. Marvit, and S. L. C. Woo, "Molecular structure and polymorphic map of the human phenylalanine hydroxylase gene," Biochemistry, vol. 25, no. 4, pp. 743-749, 1986.

[12] M. A. Cleary, "Phenylketonuria," Paediatrics and Child Health (United Kingdom), vol. 25, no. 3, pp. 108-112, 2015.

[13] D. H. Lee, S. K. Koo, K.-S. Lee et al., "The molecular basis of phenylketonuria in Koreans," Journal of Human Genetics, vol. 49, no. 11, pp. 617-621, 2004.

[14] C. R. Scriver, "Hyperphenylalaninemia: phenylalanine hydroxylase deficiency," in The Metabolic and Molecular Bases of Inherited Disease, C. R. Scriver, A. L. Beaudet, W. S. Sly et al., Eds., vol. 110, pp. 205-206, McGrawHill, New York, NY, USA, 8th edition, 2001.

[15] H. Al-Hosani, M. Salah, D. Saade, H. Osman, and J. AlZahid, "United Arab Emirates National Newborn Screening Programme: An evaluation 1998-2000," Eastern Mediterranean Health Journal, vol. 9, no. 3, pp. 324-332, 2003.

[16] H. Al Hosani, M. Salah, H. M. Osman et al., "Expanding the comprehensive national neonatal screening programme in the United Arab Emirates from 1995 to 2011/Extension du vaste programme national de depistage neonatal aux Emirats arabes unis de 1995 a 2011," Eastern Mediterranean Health Journal, vol. 20, no. 1, p. 17, 2014.

[17] F. A. Al-Jasmi, A. Al-Shamsi, J. L. Hertecant, S. M. AlHamad, and A. Souid, "Inborn errors of metabolism in the United Arab Emirates: disorders detected by newborn screening (2011-2014)," in JIMD Reports, vol. 28, pp. 127-135, Springer, Berlin, Germany, 2016.

[18] I. Özalp, T. Coşkun, A. Tokatli et al., "Neonatal PKU screening in Turkey: 7 years experience in a developing country," Screening, vol. 4, no. 3, pp. 139-147, 1995.

[19] A. N. K. Abu Shahla, Y. Abed, and N. K. Abu Shahla, "Screening programme for phenylketonuria in the Gaza Strip: Evaluation and recommendations," Journal of Tropical Pediatrics, vol. 50, no. 2, pp. 101-105, 2004.

[20] A. M. Hamawandi, A. P. Rashid, H. H. Saeed, and O. M. Hawrami, "Annual incidence of phenylketonuria in Sulaimani City. Mirt Research," Journals of Medicine and Medical Sciences, vol. 3, no. 9, pp. 427-431, 2015.

[21] A. A. Lujain and A. A. Hassan, "Overview of neonatal screening program applied at primary health care centers in Baghdad/Iraq," International Journal of Community and Cooperative Studies, vol. 4, no. 2, pp. 40-56, 2016.

[22] S. Senemar, H. Ganjekarimi, M. Fathzadeh, S. Senemar, B. Tarami, and M. Bazrgar, "Epidemiological and clinical study of phenylketonuria (PKU) disease in the national screening program of neonates, Fars Province, Southern Iran," Iranian Journal of Public Health, vol. 38, no. 2, pp. 58-64, 2009.

[23] A. Habib, M. H. Fallahzadeh, H. R. Kazeroni, and A. H. Ganjkarimi, "Incidence of phenylketonuria in Southern Iran," Iranian Journal of Medical Sciences, vol. 35, no. 2, pp. 137-139, 2010.

[24] A. Abbaskhanian, D. Zamanfar, P. Afshar et al., "Incidence of Neonatal Hyperphenylalaninemia based on high-performance liquid chromatography confirmatory technique in Mazandaran Province, Northern Iran (2007-2015)," International Journal of Preventive Medicine, vol. 8, p. 39, 2017.

[25] H. Moammar, G. Cheriyan, R. Mathew, and N. Al-Sannaa, "Incidence and patterns of inborn errors of metabolism in the eastern province of saudi arabia, 1983-2008," Annals of Saudi Medicine, vol. 30, no. 4, pp. 271-336, 2010.

[26] H. El Araby, E. Fateen, and A. Gouda, "Screening for phenylketonuria and galactosemia among Egyptian newborns in Menoufiya governorate," Egyptian Journal of Medical Human Genetics, vol. 10, no. 2, 2009.

[27] I. Özalp, T. Coşkun, M. Ceyhan et al., "Incidence of phenylketonuria and hyperphenylalaninaemia in a sample of the Turkish newborn population," Journal of Inherited Metabolic Disease, vol. 9, no. 2, pp. 237-239, 1986.

[28] R. Daher, M. Beaini, R. Mahfouz, N. Cortas, and K. A. Younis, "A neonatal screening in Lebanon: Results of five years' experience," Annals of Saudi Medicine, vol. 23, no. 1-2, pp. 16-19, 2003.

[29] I. Khneisser, S. Adib, S. Assaad, A. Megarbane, and P. E. Karam, "Cost-benefit analysis: Newborn screening for inborn errors of metabolism in Lebanon," Journal of Medical Screening, vol. 22, no. 4, pp. 182-186, 2015.

[30] D. D. Farhud and M. Kabiri, "Incidence of phenylketonuria (PKU) in Iran," The Indian Journal of Pediatrics, vol. 49, no. 400, pp. 685-688, 1982.

[31] J. Golbahar, E. A. Al-Jishi, D. D. Altayab, E. Carreon, M. Bakhiet, and H. Alkhayyat, "Selective newborn screening of inborn errors of amino acids, organic acids and fatty acids metabolism in the Kingdom of Bahrain," Molecular Genetics and Metabolism, vol. 110, no. 1-2, pp. 98-101, 2013.

[32] G. C. Yadav and P. C. Reavey, "Aminoacidopathies: a review of 3 years' experience of investigations in a Kuwait hospital," Journal of Inherited Metabolic Disease, vol. 11, no. 3, pp. 277-284, 1988.

[33] S. Al Riyami, M. Al Maney, S. N. Joshi, and R. Bayoumi, "Detection of inborn errors of metabolism using tandem mass spectrometry among high-risk Omani patients," Oman Medical Journal, vol. 27, no. 6, pp. 482-485, 2012.

[34] L. A. Selim, S. A.-H. Hassan, F. Salem et al., "Selective screening for inborn errors of metabolism by tandem mass spectrometry 
in Egyptian children: a 5year report," Clinical Biochemistry, vol. 47, no. 9, pp. 823-828, 2014.

[35] W. Amayreh, A. Al-Hawamdeh, and K. Al-Qa'qa, "Spectrum of inborn errors of metabolism in jordan: five years' experience at king hussein medical center," Journal of the Royal Medical Services, vol. 19, no. 4, pp. 37-41, 2012.

[36] P. E. Karam, M.-Z. Habbal, M. A. Mikati, G. E. Zaatari, N. K. Cortas, and R. T. Daher, "Diagnostic challenges of aminoacidopathies and organic acidemias in a developing country: a twelve-year experience," Clinical Biochemistry, vol. 46, no. 18, pp. 1787-1792, 2013.

[37] R. F. Thijeel, "The prevalence of phenylkeonuria and other metabolic diseases in sick Iraqi children; the importance of the newborn screening program," Journal of the Faculty of Medicine Baghdad University, vol. 55, no. 3, pp. 214-218, 2013.

[38] S. Hala, R. F. T. Arif, and A. Farhan, "Inborn errors of metabolism status in Iraq," IOSR Journal of Pharmacy and Biological Sciences, vol. 11, no. 2, pp. 58-62, 2016.

[39] J. Golbahar, Z. Karamizadeh, and Z. Honardar, "Selective screening of amino acid disorders in the south-west of Iran, Shiraz," Journal of Inherited Metabolic Disease, vol. 25, no. 6, pp. 519-521, 2002.

[40] M. Abdel-Hamid, K. Tisocki, L. Sharaf, and D. Ramadan, "Development, validation and application of tandem mass spectrometry for screening of inborn metabolic disorders in Kuwaiti infants," Medical Principles and Practice, vol. 16, no. 3, pp. 215-221, 2007.

[41] F. Hassan, I. Aris, K. N. Zainuddin, and N. A. Alina, "Torque and power of CNGDI engine with two different piston crown shapes," Journal of Applied Sciences Research, vol. 5, no. 8, pp. 949-954, 2009.

[42] F. A. Hassan, F. El-Mougy, S. A. Sharaf et al., "Inborn errors of metabolism detectable by tandem mass spectrometry in Egypt: the first newborn screening pilot study," Journal of Medical Screening, vol. 23, no. 3, pp. 124-129, 2016.

[43] I. Ozalp, T. Coskun, S. Tokol, G. Demircin, and E. Monch, "Inherited metabolic disorders in Turkey," Journal of Inherited Metabolic Disease, vol. 13, no. 5, pp. 732-738, 1990.

[44] A. S. Teebi, S. A. Al-Awadi, T. I. Farag, K. K. Naguib, and M. Y. El-Khalifa, "Phenylketonuria in Kuwait and Arab countries," European Journal of Pediatrics, vol. 146, no. 1, pp. 59-60, 1987.

[45] S. Vallian, E. Barahimi, and H. Moeini, "Phenylketonuria in Iranian population: a study in institutions for mentally retarded in Isfahan," Mutation Research-Fundamental and Molecular Mechanisms of Mutagenesis, vol. 526, no. 1-2, pp. 45-52, 2003.

[46] N. M. Ghiasvand, A. Aledavood, R. Ghiasvand et al., "Prevalence of classical phenylketonuria in mentally retarded individuals in Iran," Journal of Inherited Metabolic Disease, vol. 32, no. 1, pp. S283-S287, 2009.

[47] B. L. Therrell, C. D. Padilla, J. G. Loeber et al., "Current status of newborn screening worldwide, 2015," Semin Perinatol, vol. 39, no. 3, pp. 171-187, 2015.

[48] American Academy of Pediatrics, "Update of newborn screening and therapy for congenital hypothyroidism," Pediatrics, vol. 117, no. 6, pp. 2290-2303, 2006.

[49] H. Bickel, C. Bachmann, R. Beckers et al., "Neonatal mass screening for metabolic disorders-summary of recent sessions of the Committee of Experts to study Inborn Metabolic Diseases, Public Health Committee, Council of Europe," European Journal of Pediatrics, vol. 137, no. 2, pp. 133-139, 1981. 


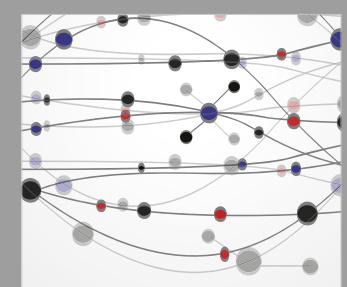

The Scientific World Journal
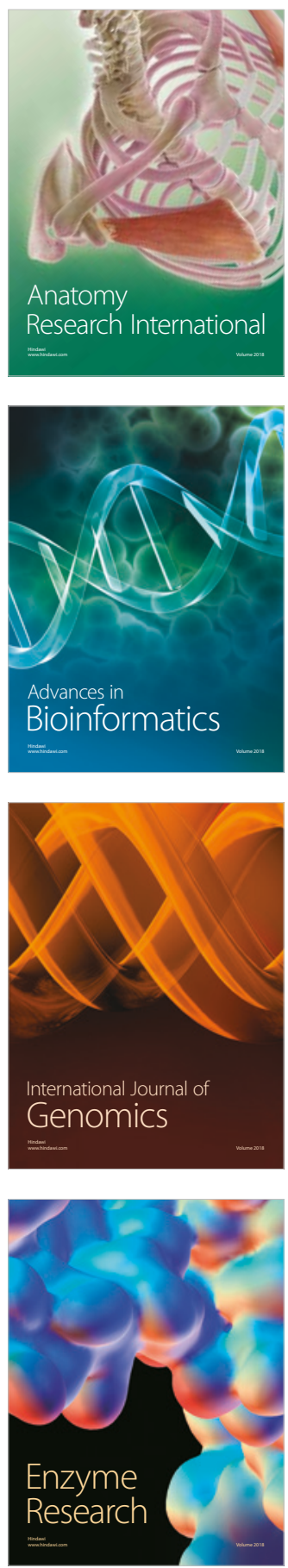
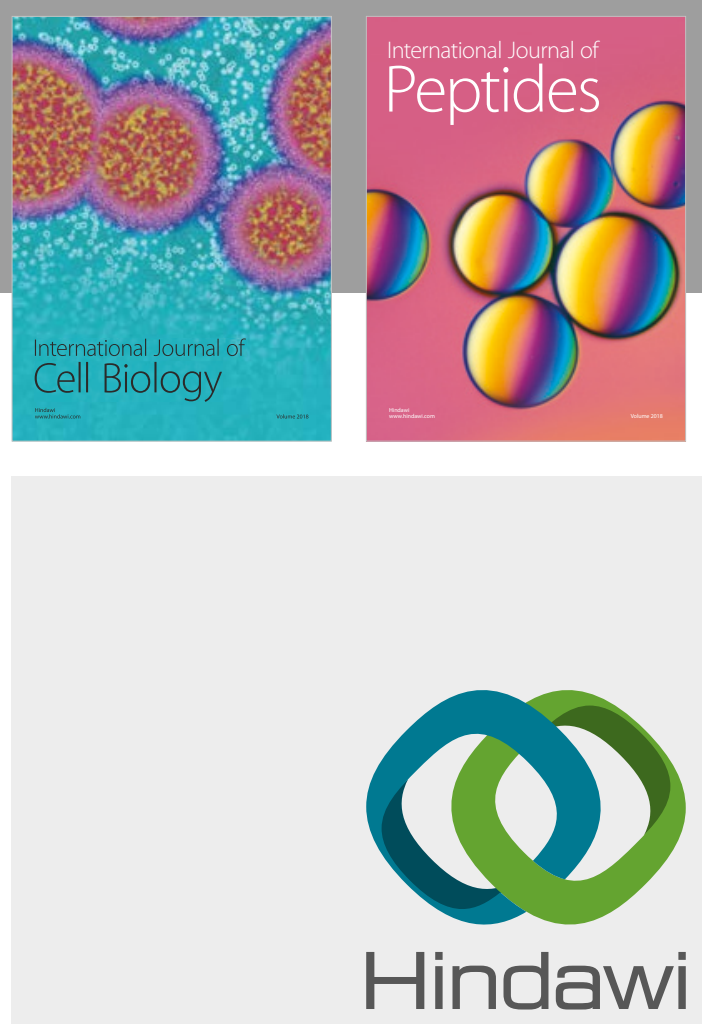

Submit your manuscripts at

www.hindawi.com
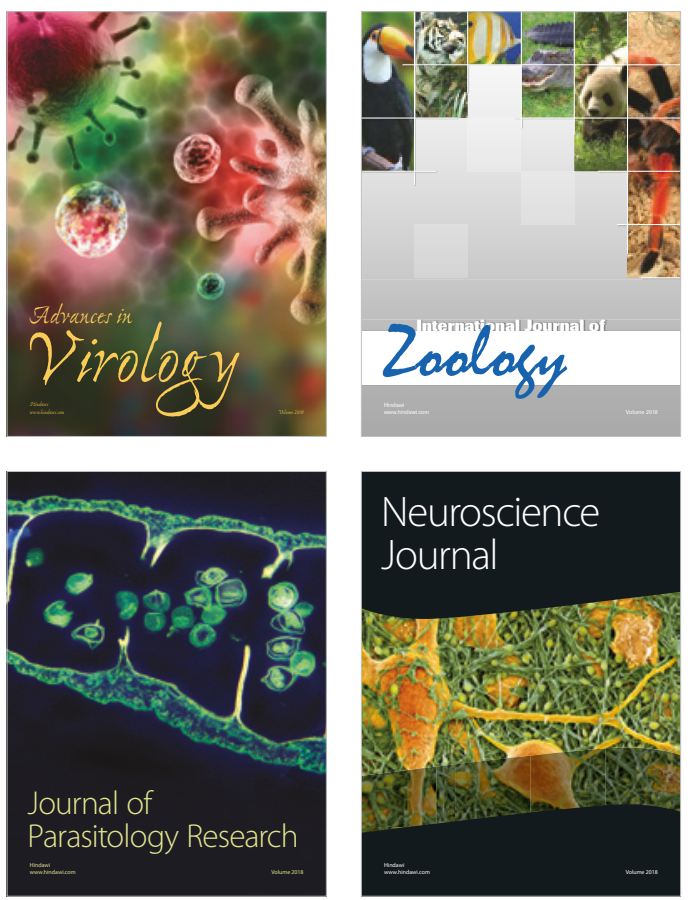
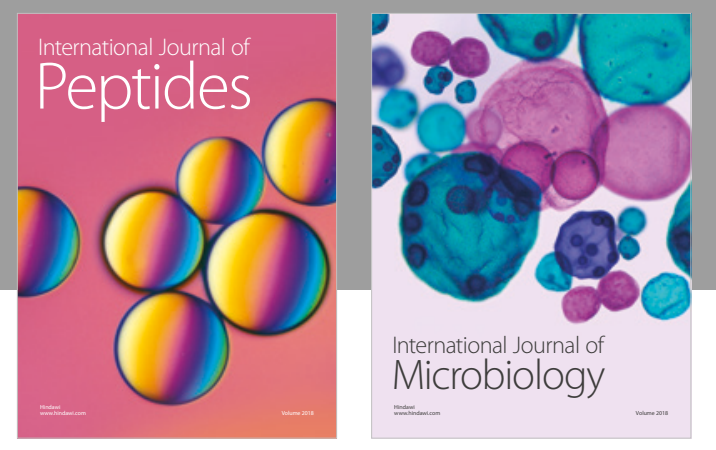

nternational Journal of Microbiology
Journal of
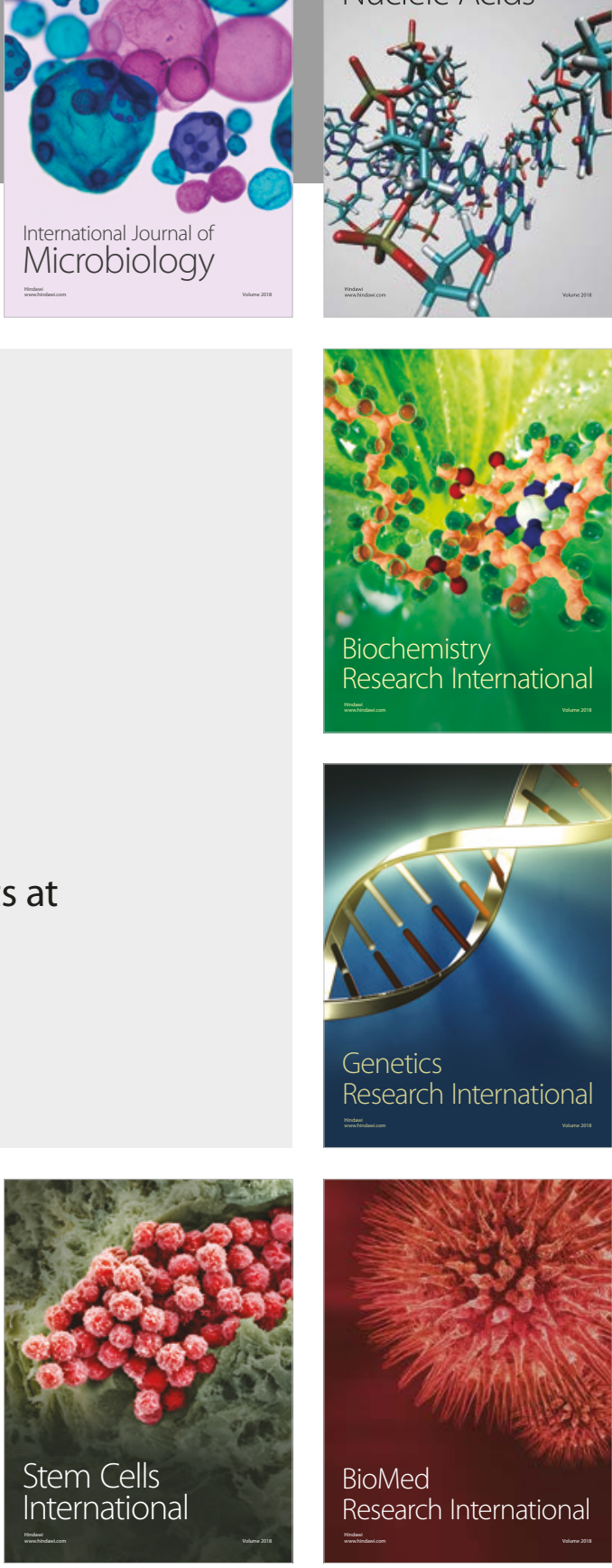
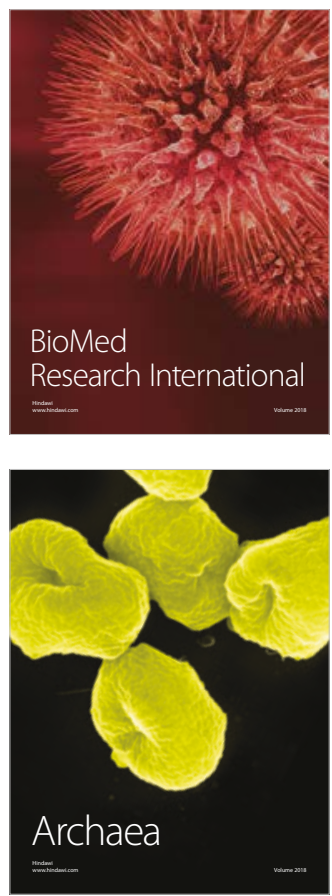